Las uniones «tempranas» en Chiapas, México

Reflexiones sobre un proceso de investigación-acción participativa (IAP) dirigida por jóvenes indígenas

Diana Reartes

Revista Argentina de Estudios de Juventud, (14), e035, 2020

ISSN 1852-4907 | https://doi.org/10.24215/18524907e035

https://perio.unlp.edu.ar/ojs/index.php/revistadejuventud

FPyCS | Universidad Nacional de La Plata

La Plata | Buenos Aires | Argentina

\title{
LAS UNIONES «TEMPRANAS» EN CHIAPAS, MÉXICO
}

\section{REFLEXIONES SOBRE UN PROCESO \\ DE INVESTIGACIÓN-ACCIÓN PARTICIPATIVA (IAP) DIRIGIDA POR JÓVENES INDÍGENAS}

\author{
«Early» unions in Chiapas, Mexico: \\ Reflections on a Participatory Action Research Process \\ Led by Indigenous Youth
}

\section{Diana Reartes}

dianareartes@ipecal.edu.mx

https://orcid.org/0000-0002-5404-3407

Instituto de Pensamiento y Cultura en América Latina (IPECAL)

México

\section{Palabras clave}

investigación-acción participativa

jóvenes indígenas uniones tempranas

Chiapas

\section{Keywords}

participatory action research

indigenous youth early unions

Chiapas

\section{Resumen}

La autora aborda en este artículo una experiencia de investigación-acción participativa (IAP) dirigida por jóvenes en tres contextos indígenas de Chiapas, México. Su principal objetivo fue documentar la configuración actual de las uniones «tempranas», aquellas que suceden cuando uno o ambos miembros de la pareja son menores de 18 años. El interés es visibilizar las dimensiones clave de estas uniones (en un contexto y tiempo determinado) así como el papel de los/as jóvenes indígenas como sujetos activos en la construcción social de conocimiento local.

\section{Abstract}

In this article the authoress addresses a recent experience of participatory action research (IAP) led by indigenous youth in three indigenous contexts of Chiapas Mexico. It is reflects on this research experience whose main documentary objective was the current configuration of the "early" unions, that is, those that happen when one or both members of the couple are under 18 years old. It is interesting to make visible the key dimensions of these unions (in a given context and time) as well as the role of indigenous youth as active subjects in the social construction of local knowledge. 


\title{
LAS UNIONES «TEMPRANAS» EN CHIAPAS, MÉXICO
}

\author{
Reflexiones sobre un proceso de investigación-acción \\ participativa (IAP) dirigida por jóvenes indígenas
}

\author{
Por Diana Reartes
}

\section{Introducción}

Por uniones «tempranas» consideramos las uniones formales o informales en las que al menos uno de los contrayentes es menor de 18 años. Utilizamos este concepto, y entrecomillamos tempranas, a sabiendas de que en los contextos estudiados esta práctica se encuentra generalizada en la población y así lo ha sido en las últimas décadas.

Según UNICEF (2019), diez países cuentan con los más altos números absolutos de matrimonio infantil, tomando como población las mujeres de entre 20 y 24 años que se casaron antes de cumplir los 18 años. India ocupa el primer lugar con 26.610.000, seguido por Bangladesh (3.931.000) y por Nigeria (3.306.000). Entre las causas de este problema se encuentran las desigualdades de género ancladas en un sistema patriarcal de poder y de subordinación, ciertas prácticas sociales y culturales, y la pobreza y la inseguridad que existen en determinados contextos marcados por la violencia, los conflictos armados o los desastres naturales. Las implicaciones son múltiples y se manifiestan en el corto, mediano y largo plazo, en el ámbito educativo, de salud física y emocional, y de bienestar psicosocial.

Los matrimonios infantiles y las uniones «tempranas» han recibido una atención limitada y reciente en América Latina y el Caribe. En 2017, en toda la región, 23\% de las mujeres de entre 20 y 24 años ya había estado casada o en unión a los 18 años, y $5 \%$ a los 15 años. Los datos sobre tendencias muestran que se trata de la única región del mundo donde no se ha registrado un descenso significativo en los últimos 
veinte años. Los países con mayor prevalencia son República Dominicana y Brasil (36\%), Nicaragua (35\%), Honduras (34\%), Guatemala (30\%), y el Salvador y México (26\%) (Greene, 2019).

La invisibilidad del fenómeno se debe, en parte, al arraigo histórico y cultural a y su naturalización, y se ve reforzada por la falta de datos a largo plazo y porque la información básica sobre matrimonio infantil no se actualiza o no está disponible en algunos países (Greene, 2019, p. 8).

Otro aspecto que afecta el estudio de la problemática y la comparación entre diferentes regiones, países y contextos se relaciona con el uso de diversas expresiones para designar este fenómeno: unión consensual, unión forzada, unión temprana, matrimonio infantil, unión libre, unión de hecho. Asimismo, el término unión se utiliza para referir tanto a casarse como o juntarse (Greene, 2019).

México presenta la quinta tasa más alta de América Latina y el Caribe, estimándose que $22,9 \%$ de las mujeres de entre 20 y 24 años se casó o entró en una unión informal antes de los 18 años, y esta tasa no ha cambiado en casi treinta años (Rivera \& Palma, 2017). En Chiapas, la tasa de matrimonio infantil alcanza un nivel de 30\%, y es también la tasa para la población rural en 14 Estados (Pérez Amador \& Hernández, 2015, citado en Rivera \& Palma, 2017, p. 4). Si bien la Ley General de Derechos de Niñas, Niños y Adolescentes (2014) establece los 18 años como edad mínima para contraer matrimonio, los códigos civiles y de familia de algunos Estados y el Código Civil Federal permiten el matrimonio infantil a través de dispensas o de excepciones.

En los objetivos de Desarrollo Sostenible (ODS), el referido a «lgualdad de género» plantea una meta específica para prácticas tradicionales nocivas, que incluyen el matrimonio infantil, y como parte de la Iniciativa 18+ en América Latina y el Caribe se han impulsado estudios que buscan destacar las especificidades de la región y encontrar similitudes y diferencias que permitan informar mejor las políticas públicas (PNUD, 2020).

En México, el estudio de las prácticas matrimoniales en contextos rurales e indígenas cuenta con una larga trayectoria, en tanto la antropología siempre ha tenido como interés el estudio del parentesco y la búsqueda de modelos 
y de patrones en lo que respecta, por ejemplo, al tipo de arreglos y a los rituales asociados (González Montes, 1999; González Montes \& Mojarro Iñiguez, 2011; Mindek, 2003).

Solo en los últimos años, la mirada y el abordaje teórico y metodológico de la perspectiva de género ha complejizado su estudio y ha comenzado a visibilizar el papel del patriarcado y la subordinación femenina en la conformación de las uniones. Por otro lado, el reciente impulso global y regional proveniente de distintos organismos internacionales y nacionales ha permitido poner en agenda la importancia de estudiar y de intervenir para prevenir, disminuir y erradicar esta práctica violatoria, y para garantizar los derechos de niñas, adolescentes y jóvenes.

Las transformaciones que se observan en los contextos rurales e indígenas adquieren una particular complejidad por cuanto en su interior (tanto en términos materiales como discursivos) coexisten prácticas y discursos «heterogéneos, fragmentarios y contradictorios» (Valdés, Benavente \& Gysling, 1999, p. 12), en relación con las relaciones entre mujeres y varones, la maternidad, la paternidad y la autonomía de los sujetos para decidir sobre su vida sexual y reproductiva. Existen sectores y contextos más permeables a los cambios y a las transformaciones, y otros más conservadores, donde continúan vigentes normas más rígidas que modelan los comportamientos sexuales, la vida en pareja y familiar, y la relación de los padres con los hijos (Valdés, Benavente \& Gysling, 1999).

Tanto la sexualidad como la reproducción, aspectos centrales al estudiar las uniones tempranas, están moldeadas por el contexto sociocultural en el que ocurren y son influidas por sus transformaciones (Oliveira \& Salles, 1986, en Salles \& Tuirán, 1997). En tanto fenómenos construidos socialmente, dichas prácticas están atravesadas por significados que son diversos en función de las múltiples adscripciones identitarias que constituyen a los sujetos. Estos significados, además, están moldeados por ciertas instituciones, lo que no invalida que las normas y las reglas hegemónicas sean resignificadas, resistidas o impugnadas por los conjuntos sociales.

En el ámbito de la formación de las parejas, sigue vigente el peso de las instituciones clásicas -la familia, la escuela, las iglesias, la biomedicina y la ley-, en la vigilancia y en el control de los comportamientos sexuales, esto dependiendo de los contextos sociohistóricos así como de los sectores sociales involucrados. 
Al estar conformados por diversos ejes de desigualdades, los procesos de formación y de disolución de uniones tempranas resultan un campo relevante para el análisis empírico de la «interseccionalidad». Las clasificaciones de género, clase, etnicidad, edad, condición migratoria e inserción laboral emergen como clasificaciones sociales principales que producen interseccionalmente diferentes trayectorias y experiencias en la vida cotidiana de los/as jóvenes, lo que influye de manera directa en situaciones de desigualdad, de exclusión, de discriminación y de vulnerabilidad (Magliano, 2015).

En otras palabras, la perspectiva interseccional nos permite observar cómo las posibilidades y las opciones que se les presentan a las mujeres y a los varones al momento de unirse son diferenciales según la posición que ocupan en sus lugares de origen, las expectativas familiares, la dinámica familiar y comunitaria, las experiencias juveniles y sus proyectos de vida.

Algunos estudios, acertadamente, han favorecido el desarrollo de investigaciones lideradas por los/as propios/as jóvenes. Las reflexiones que se presentan a continuación se derivan de una investigación-acción participativa (IAP) llevada a cabo por la organización civil Ch’ieltik (Ideas, Información y Diseños Educativos para

Acciones Saludables A.C.), ${ }^{1}$ con el financiamiento del Fondo para Jóvenes de Centroamérica y México (CAMY). Dicha experiencia se desarrolló entre febrero de 2018 y junio de 2019, y quien esto escribe facilitó y coordinó el proceso desarrollado por un equipo de seis jóvenes originarios de las comunidades en las que se realizó el trabajo de campo. ${ }^{2}$

\section{Los objetivos de la IAP}

- Documentar cómo se configuran las uniones tempranas en tres contextos indígenas: San Juan Cancuc, Ejido Veracruz y Nuevo San Juan Chamula, a partir de explorar causas, dimensiones problemáticas y consecuencias.

- Lograr una aproximación al conocimiento sobre los cambios y las continuidades en torno a las uniones tempranas.

— Formar a jóvenes hablantes de lenguas indígenas, originarios de estas comunidades, en proyectos de investigación-acción participativa. 
— Señalar recomendaciones destinadas al diseño de estrategias educativas adecuadas culturalmente para prevenir las uniones tempranas y para atender algunas de sus dimensiones problemáticas.

\section{Los contextos estudiados}

Ubicado en el sureste mexicano, Chiapas es el Estado con mayor porcentaje de pobreza (76.2\%), según el Programa de las Naciones Unidas para el Desarrollo (PNUD, 2014). Para 2015, contaba con 5.217.908 habitantes. De esta población, $49 \%$ es urbana, $51 \%$ es rural y $27,2 \%$ habla una lengua indígena (INEGI, 2010). De acuerdo a los indicadores de carencias sociales, $30 \%$ de la población tenía rezago educativo, $81,2 \%$ no poseía acceso a seguridad social y 54,6\% carecía de acceso a servicios básicos en la vivienda (CONEVAL, 2015).

La elección de los municipios indígenas en los que se llevó a cabo la investigación tuvo en cuenta el trabajo previo de la organización civil con jóvenes de estas localidades. Se trabajó en tres municipios: San Juan Cancuc, en la Región Altos, y Ejido Veracruz y Nuevo San Juan Chamula, en la región Selva, todos ellos con un alto grado de marginación y pobreza. En Cancuc, por ejemplo, la pobreza alcanza 97,3\% (PNUD, 2014). Con un promedio de 900 habitantes, el índice de fecundidad promedio es de 3,5 hijos por mujer, la escolaridad promedio es de cuatro años y existe pluralidad de cultos (iglesias católicas, evangélicas, protestantes y presbiterianas). La migración interna e internacional está presente, particularmente, en la población juvenil; predomina la producción agrícola, cafetalera, porcina y artesanal; la herencia es por vía masculina y la residencia posunión es patrivirolocal.

En los últimos veinte años, distintos tipos de fenómenos han impactado en las familias rurales, en su organización y en su dinámica. Por un lado, están los cambios económicos que afectaron las actividades agropecuarias:

El cambio de modelo económico y la crisis de 1994 afectaron la viabilidad de las actividades agropecuarias [... ] de los productores campesinos de pequeña escala [...] situación que obligó a la gente [...] a buscar trabajo e ingresos fuera y lejos de las comunidades de origen de manera prolongada si no es que indefinida (Arias, 2014, p. 183). 
Por el otro, están los cambios demográficos, entre cuyas consecuencias destacan:

[...] la reducción del tamaño de la familia, el aumento en la esperanza de vida de los hombres y sobre todo de las mujeres, el incremento de la escolaridad, el envejecimiento de la población rural, el aumento de la edad de matrimonio, la reducción de la tasa de fecundidad [...], el aumento de las uniones consensuales, la creciente inestabilidad de las uniones, la formación de hogares independientes encabezados por mujeres que han decido salir de la violencia doméstica (García Oliveira, 2011; González, 2007; Navarro, 2010, citados en Arias, 2014, p. 183).

\section{Puntos de partida}

\section{La investigación-acción participativa dirigida por jóvenes}

El proyecto se planteó como una investigación-acción participativa (IAP) dirigida por jóvenes indígenas interesados/as en promover acciones transformadoras con pares en sus comunidades de origen. Se concibió como un proceso de indagación y de reflexión orientado a lograr una aproximación a una situación/problema específico para comprender sus dinámicas y las personas involucradas; enfoque metodológico que ha tenido un gran crecimiento en los últimos años para investigar cuestiones de salud pública, de educación y de psicología comunitaria (Ozer, 2017, p. 174).

En primer lugar, la IAP se nutre de aproximaciones metodológicas conocidas como investigación-acción, investigación participativa, investigación colaborativa y coinvestigación que, en términos comunes, se distancian de «las dinámicas y las relaciones de poder encarnadas en la investigación tradicional» y asumen el desafío de establecer relaciones más horizontales entre quienes «investigan» y quienes son «investigados» (Dietz \& Álvarez Veinguer, 2014, p. 61).

En términos gnoseológicos, este tipo de investigación tiene como objetivo

[...] superar la separación entre sujeto y objeto. Aquellos afectados por el problema son la fuente primaria de información y los actores principales en la generación, la validación y el uso del conocimiento para la acción. El investigador es parte de la comunidad afectada (Loewenson, Laurell \& Hogstedt, 1994, citado en Loewenson y otros, 2014, p. 12). 
Por otra parte, esta aproximación «reconoce la riqueza de los recursos que los miembros de la comunidad aportan a los procesos de conocer, de crear conocimiento y de actuar sobre ese conocimiento para lograr un cambio» (Loewenson y otros, 2014, p. 12).

Dentro de la investigación social sobre juventud y desarrollo, varios enfoques metodológicos, denominados Youth-led Particpatory Action Research (YPAR), Youthled Research, Evaluation and Planning (Youth REP) y Youth-led Research, tienen en común «[...] empoderar a niños, adolescentes y jóvenes que crecen en circunstancias difíciles, para entender mejor sus comunidades y sus características y los valores sociales que los afectan para que lleguen a ser agentes de cambio activo» (ICDI, 2019, p. 5, traducción de la autora del artículo). ${ }^{3}$

Esto conlleva ofrecer a los/as jóvenes el acompañamiento de un adulto facilitador que comparta con ellos/as recursos y herramientas tendientes a identificar y a analizar problemas relevantes en sus vidas, a partir de elaborar el diseño de la investigación y de seleccionar los métodos para utilizar información existente o para generar nueva información desde la comunidad (Ozer, 2017, p. 174).

De este modo, son los/as jóvenes quienes lideran el proceso investigativo, acompañados por un/a investigador/a social que tiene el papel de facilitar los «métodos y los procesos organizados que permitieron a aquellos involucrados de forma directa compartir, analizar y validar su experiencia colectivamente. Esto significa que la reflexión, la interpretación y el conocimiento generados se mantienen dentro de la comunidad en el proceso» (Loewenson y otros, 2014, p. 24).

Dicha aproximación presenta como objetivos: «1. Apoyar a las y los jóvenes a entender el mundo en el que viven, 2. Dotarlos de las habilidades necesarias para tomar decisiones, 3. Apoyarlos para convertirse en agentes de cambio en sus comunidades» (ICDI, 2019, p. 6, traducción de la autora del artículo). ${ }^{4}$ Para los/as jóvenes, los beneficios suponen: «Mejorar la capacidad para realizar elecciones, reflexionar y pensar críticamente; mejorar la comunicación escrita y las habilidades de organización; mayor confianza para contribuir a mejorar la vida de los adolescentes y jóvenes en sus comunidades; obtener valiosos conocimientos y contribuciones originales al conocimiento local; y observar con ojos diferentes, preguntar por diferentes asuntos, tener diferentes preocupaciones» (ICDI, 2019, p. 6, traducción de la autora del artículo). ${ }^{5}$ 
Una característica de esta aproximación es que intenta establecer entre los/as participantes relaciones lo más horizontales posibles, a partir de intercambios comunicativos tendientes a generar conocimientos grupales, aprendizajes mutuos, procesos de reflexión colectiva, y enriquecimiento individual y grupal. En este sentido, uno de los principios que actuó como eje fue el establecimiento de relaciones recíprocas entre los/as integrantes del equipo de investigación, mediante la inclusión de las voces de todos/as los/as participantes en los momentos clave del proceso de decisión: definir objetivos del estudio, conceptos clave y métodos de indagación, recolección de datos y análisis e interpretación de los resultados (Riaño, 2012).

Según Emily Ozer (2017), se trata de una aproximación innovadora para conducir las preguntas científicas, que involucra a los/as jóvenes como expertos/as y como coinvestigadores/as y que modifica los supuestos estándar acerca de quién es el experto para crear conocimiento acerca de la gente joven. La autora también señala un conjunto de efectos positivos para los/as participantes: el incremento en su autonomía psicosocial; el propósito y el rol de la responsabilidad; y el fomento de un sentido de identidad colectiva, particularmente importante para los/as jóvenes marginados/as como resultado de su condición étnica, inmigración, clase social u orientación sexual. De igual modo, se identifica el fortalecimiento de «relaciones de apoyo y de cooperación entre jóvenes y adultos»(Ozer, 2017, p. 175). Es importante señalar, también, que este tipo de investigaciones promueven «la conciencia crítica para ir más allá de las explicaciones de los problemas a nivel individual [...] para investigar y trabajar en factores sistémicos» (Ozer, 2017, p. 176).

Por último, a nivel de las contribuciones en el campo del desarrollo, la IAP permite a los/as jóvenes investigadores/as estudiar problemáticas sensibles como las relaciones sexuales, el bulling o la violencia juvenil, lo que favorece investigaciones que se enriquecen con la mirada, las preguntas y los métodos de indagación de los/as jóvenes investigadores. Pero, tal vez, lo más importante es que esta perspectiva cambia las normas acerca de quién crea conocimiento y la mirada con que el mundo adulto ve a los/as jóvenes, de percibirlos/as como problema los pasa a considerar como expertos/as que pueden generar resultados científicos para informar acciones (Ozer, 2017, p. 176). 
En otras palabras, al adoptar esta metodología se buscó que los/as participantes lograran:

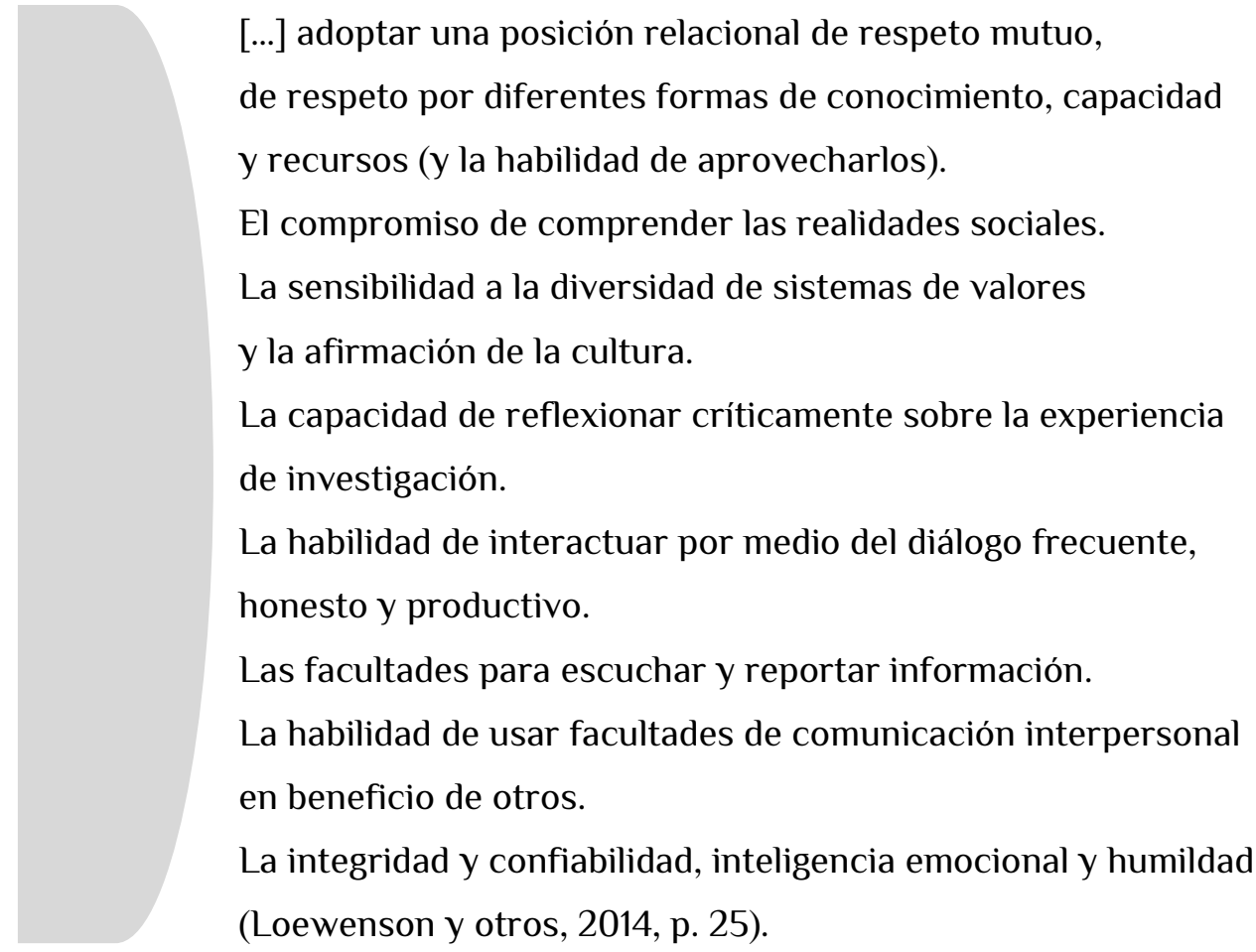

Los momentos de la IAP sobre uniones «tempranas»

El proceso llevado a cabo incluyó los siguientes momentos:

\section{a) Convocatoria y selección de los/as jóvenes}

El proyecto convocó a jóvenes mujeres y varones, hablantes de lenguas indígenas, con interés en problemáticas juveniles, con experiencia en trabajo comunitario y con estudios de nivel medio superior (en varios casos, universitarios). Se seleccionaron con este fin un grupo de seis investigadores/as (tres mujeres y tres varones), habitantes de las localidades donde realizamos el trabajo de campo y con lazos de pertenencia y de afinidad con la comunidad y la población juvenil. Una de las jóvenes fungió como coordinadora logística. 


\section{b) Momento de capacitación y de entrenamiento}

Mi papel fue el de acompañar el proceso mediante tres talleres presenciales, reuniones semanales de retroalimentación del trabajo con el equipo, y evaluación colectiva de los avances y las dificultades que se presentaron a lo largo del proceso.

Los talleres impartidos al comienzo, durante y al final del proceso capacitaron a los/as jóvenes en diversos aspectos sobre cómo llevar a cabo una investigaciónacción-participativa organizados en torno a los siguientes tópicos:

- Características de la IAP.

- Pasos en el diseño de la IAP (diagnóstico, planificación, ejecución, sistematización y análisis).

— Técnicas para identificar problemas comunitarios, sus causas y sus efectos.

n Formulación de preguntas y de objetivos de investigación.

- Instrumentos de recolección de información primaria y secundaria.

- Selección de entrevistados/as (criterios de selección y cómo evitar sesgos).

n Pautas para trabajar colectivamente y con los/as entrevistados.

- Ética en la IAP.

- Análisis de información cualitativa (convergencias

y divergencias en las percepciones de los/as entrevistados/as.

• Producción de un reporte de investigación.

— Búsqueda de estrategias para afrontar la problemática.

- Difusión de resultados: formatos, audiencias.

— Evaluación y sistematización de la experiencia de IAP.

Cabe mencionar que cada viernes, durante todo el tiempo que duró la investigación, el equipo mantuvo reuniones por whatsapp para conversar sobre los avances y las dificultades que se iban presentando en las actividades realizadas y para esclarecer dudas. La facilitadora supervisó las entregas por escrito de cada uno/a de los/as integrantes y realizó devoluciones en forma personal y grupal. 


\section{c) Recolección de datos primarios}

En un primer momento, incluyó visitas a las comunidades e interlocución con autoridades locales para dar a conocer el proyecto y para obtener la autorización correspondiente. En un segundo momento, se establecieron conversaciones informales con abuelos/as o con padres/madres de los/as jóvenes del equipo sobre su propia experiencia en uniones «tempranas».

A posteriori, se contactaron jóvenes (mujeres y varones) que se hubieran unido antes de los 18 años y que desearan compartir su experiencia. Luego de una primera selección, varios/as jóvenes que habían manifestado su interés desertaron o, como en el caso de algunos varones, no estaban disponibles porque iban a migrar. Los jóvenes indígenas se contactaron atendiendo a las siguientes características: mujeres y varones que se hayan unido en los últimos cinco años, antes de los 18 años, con y sin hijos/as, con distinto nivel educativo y diferentes inserciones laborales. El acercamiento y la selección de los/as entrevistados/as tuvo en cuenta el conocimiento de los/as investigadores/as sobre los/as jóvenes que habían transitado por esta experiencia en su comunidad.

En un tercer momento, se entrevistaron los/as jóvenes, actividad que duró cuatro meses en promedio. En San Juan Cancuc, se entrevistó a 12 jóvenes; en los dos municipios restantes, a 10 jóvenes (mitad mujeres y mitad varones). En total, se recogieron relatos de 32 jóvenes. Como principal técnica de investigación para captar el punto de vista de los sujetos involucrados, se utilizó la entrevista individual estructurada, a través de la cual se obtuvieron verbalizaciones, enunciados y narraciones en función de determinados ejes temáticos, los que, sin embargo, se concibieron como flexibles para favorecer la emergencia y el abordaje de aspectos no contemplados y relevantes para los/as entrevistados/as. La participación de los/as jóvenes investigadores/as favoreció la elaboración de guiones con un lenguaje entendible y accesible. El guion de la entrevista fue elaborado en español e interpretado al tsotsil, tseltal, tojolabal y kanjobal. En muchos casos, el/la entrevistado/a contestó las preguntas en su lengua materna y el contenido fue interpretado al español por el/la entrevistador/a. La realización de las entrevistas implicó varios encuentros con cada entrevistado/a. 
En cada uno de los contextos, las entrevistas fueron realizadas por una pareja integrada por un varón y una mujer. Las mujeres entrevistaron a las muchachas y los varones a los muchachos. En uno de los contextos, el Ejido Veracruz, el investigador y la investigadora realizaron entrevistas a jóvenes que eran pareja, pero lo hicieron en forma separada.

\section{d) Transcripción, codificación e interpretación de entrevistas}

En algunos casos, las entrevistas fueron interpretadas a la lengua materna del/la entrevistado/a. A medida que se iba respondiendo a las preguntas, el/la entrevistador/a tomaba notas. Finalizada la entrevista, la reconstruía en un archivo electrónico y consignaba en forma de notas toda aquella información que fuera relevante al contexto de su realización. Una vez transcriptas, las entrevistas eran enviadas a la coordinadora de la investigación quien las revisaba y en las reuniones del equipo se discutía la información que iba surgiendo, los vacíos y las nuevas preguntas que intentaban aclarar o profundizar algún aspecto importante.

Una vez realizadas todas las entrevistas, se inició la tarea de codificación manual que, luego, dio paso a la etapa de análisis de la información con la intención de detectar semejanzas y diferencias entre puntos de vista, creencias y conductas de los/as jóvenes entrevistados/as en cada uno de los contextos seleccionados.

\section{e) Elaboración del informe final}

A partir de informes parciales, se elaboró colectivamente un informe final con hallazgos y con resultados, que intentó dar respuestas a las principales preguntas y objetivos, ofreciendo conclusiones y recomendaciones.

\section{f) Devolución y difusión de resultados}

La devolución de los resultados a la comunidad y a los/as jóvenes fue concebida como una exigencia ética y como parte sustancial del proceso, tendiente a visibilizar problemáticas juveniles y a promover estrategias de empoderamiento a través de propuestas de intervención. Para tal fin, se elaboraron un guion radiofónico y un video corto que se ha comenzado a difundir en las comunidades en distintas actividades culturales y educativas. 


\section{g) Sistematización y evaluación de la experiencia}

Refiere al momento en que se evaluaron los aprendizajes, los logros y los aspectos que podrían ser mejorados, desde el punto de vista individual y colectivo.

\section{Importancia de los conocimientos adquiridos}

El acercamiento a estos tres contextos indígenas nos permitió tener un panorama exploratorio del modo en que se presentan las uniones antes de los 18 años. Este proceso de indagación y de reflexión colectiva permitió explorar los condicionantes socioculturales e ideológicos que favorecen la formación de parejas jóvenes y algunas de las desigualdades sociales que los/as intersecan.

El conjunto de hallazgos y de resultados permite observar cómo las desigualdades sociales, de género, generacionales y étnicas se intersecan, lo que determina que continúe vigente «un patrón de unión temprana combinado con el inicio de la vida sexual y con un inmediato inicio de la vida reproductiva» (Solís y otros, 2008, citado por Ballinas-Urbina y otros, 2015, p. 278).

Las localidades donde trabajamos se caracterizan por la pobreza y la marginación, el rezago educativo, la inexistencia de proyectos productivos para los/as jóvenes, la falta de opciones y de facilidades para que puedan continuar sus estudios, la insuficiencia de servicios que satisfagan necesidades de información y el acceso a métodos para prevenir y/o interrumpir embarazos, y la escasa posibilidad del ejercicio de derechos en la población juvenil.

La prolongación de la vida escolar (más allá de la primaria), la migración, el acceso a medios masivos de comunicación y la labor de organizaciones civiles que trabajan a favor de la eliminación de la desigualdad de género y de las violencias, y en pos de los derechos, ha ido posibilitando dinámicas y cambios importantes en el modo en que la juventud indígena se ve a sí misma y en su relación con el mundo adulto.

El noviazgo ha emergido como una instancia anterior a la unión, particularmente para el sector escolarizado de la población juvenil indígena. Aunque en algunas comunidades todavía no es muy bien visto; en otras, es más aceptado por los padres y por la comunidad (Cruz Salazar, 2009; Reartes, 2014; Rodríguez, 2000; Rodríguez 
\& De Keijzer, 2002). Ello ha implicado enormes cambios en la subjetividad de los/as jóvenes, quienes ya consideran su derecho elegir a su pareja y poder ejercer su sexualidad antes de unirse.

La migración ha sido otra dimensión que ha favorecido que los/as jóvenes puedan iniciar relaciones de noviazgo en los lugares a los que migran, sin el control de los padres (Evangelista García \& Kauffer Michel, 2007).

Algunos de los problemas detectados que predisponen a las uniones "tempranas» son: la falta de un acceso efectivo a anticonceptivos, tanto por cuestiones ideológicas como por la falta de accesibilidad a personas o a servicios que puedan satisfacer la demanda si la hubiera; la deserción escolar; la falta de oportunidades laborales, particularmente para las mujeres, y de un proyecto de vida que vaya más allá de formar una familia y tener hijos/as; la violencia, sufrida particularmente por las jóvenes en sus familias de origen; el embarazo; los obstáculos económicos e ideológicos que imposibilitan continuar los estudios más allá de la secundaria o la preparatoria, tanto para las mujeres como para los varones; la sujeción juvenil frente a la autoridad de los padres; la mayor vulnerabilidad que enfrentan las jóvenes con hijos/as pequeños/as si se separan y regresan a sus comunidades.

En el ámbito de la conformación de las uniones, encontramos como similitudes que la mayoría de los/as jóvenes iniciaron su vida sexual con quien luego se unieron durante el periodo de noviazgo. No contaron con información significativa que posibilitara la adopción de métodos anticonceptivos durante este periodo, lo que derivó en embarazos que precipitaron la unión, en coyunturas donde los/as jóvenes deben subordinarse a las normas sociales que prescriben la unión.

La información etnográfica permite visualizar dos tipos de uniones: la unión cuando existe un embarazo y la unión sin embarazo.

Cuando existe un embarazo, las uniones se presentan como la solución a la «transgresión» de haber tenido relaciones sexuales fuera de un matrimonio y son «obligadas» por la normatividad social vigente, que impide que la maternidad pueda ser ejercida por fuera de una unión. Del lado de la mujer, la unión sirve para salvar el honor de la joven y de su familia, y legitima la maternidad. Del lado del varón, aceptar la unión habla bien de él, en tanto hacerse cargo del embarazo lo presenta como responsable de su comportamiento. 
En el caso de aquellas parejas que acuerdan unirse para formar una familia, podemos pensar que son obligadas en tanto todavía no es posible imaginar en estos contextos noviazgos más largos que les permitan desarrollar otros proyectos o la posibilidad de tener varias relaciones de noviazgo antes de decidir su unión. El iniciar una vida en pareja es una de las únicas opciones que se le presenta a la mayoría de los/as jóvenes luego de finalizar la secundaria. Esto, a pesar de las verbalizaciones de muchos/as de ellos/as que indicaron que la edad ideal para casarse se situaría más allá de los 18 años y que sería bueno prolongar el tiempo del noviazgo con el fin de conocerse más.

Las consecuencias de la unión afectan el bienestar psicosocial de la pareja y son distintas para mujeres y para varones. En primer lugar, si se abandona la escuela esto ocasiona en algunos casos frustración y decepción, porque ya no se pueden llevar a cabo ciertos proyectos vitales. Además, tanto mujeres como varones manifestaron la exigencia de tener que asumir ciertas responsabilidades para las que no se sentían preparados/as como tener que trabajar más frente a la llegada de su hijo/a. En este terreno, son los varones los que, por su papel de proveedores, se sienten más exigidos al tener que encontrar fuentes de ingresos extra.

En cuestiones de dinámica familiar, debido al cambio de residencia de las mujeres, son las jóvenes las que deben adaptarse a nuevas normas de convivencia familiar y, en ocasiones, a la conversión religiosa y a la subordinación frente a sus suegros y a su pareja. La relación con la suegra es fuente de conflictos y de violencia, y trae como consecuencias que se ponga en duda la conducta moral de la joven, su honorabilidad, su buen desempeño como esposa, todo lo cual puede conllevar tristeza, enojo, vergüenza, aislamiento y soledad.

Todo ello, incrementa la vulnerabilidad de las jóvenes y precariza su condición de mujer y madre de un recién nacido, más aún cuando la joven opta por regresar con su familia de origen para poner fin a un ambiente de hostilidad. El regresar a su comunidad de origen vuelve a colocarlas en la mira, culpabilizándolas por no ser «buenas esposas» y por ser las causantes de la disolución del vínculo.

De este modo, las normas de género y de generación colocan a las parejas jóvenes bajo la autoridad de padres y de autoridades, y subordinan a las mujeres a padres, a parejas y a suegras. Estas últimas, se constituyen en sostenedoras de la transmisión de la violencia hacia las mujeres más jóvenes de la parentela. 
Si bien en estos contextos las uniones a temprana edad aparecen normalizadas, creemos importante señalar que estas deben ser consideradas «obligadas» -tanto en los casos en los que se presenta un embarazo como en aquellos donde las parejas acuerdan iniciar la convivencia- debido a la vigencia de ciertos mandatos sociales.

En cuanto a la experiencia adquirida por el equipo de jóvenes investigadores/as a nivel individual, los/as participantes del equipo puntualizaron que los principales aprendizajes fueron:

- Reconocer los aportes personales para desarrollar un trabajo en equipo.

- Conocer las realidades de los/as jóvenes de su comunidad desde otro punto de vista y tener otra perspectiva sobre sus problemáticas.

- Trabajar con otro/as jóvenes.

n Acercarse a las personas con respeto y dialogar con ellas «sin incomodarlas»: «No solo era la entrevistadora sino también oyente y amiga, confidente, alguien que puede consolar a la otra persona».

nealizar una investigación «paso a paso».

n Aprender de las experiencias de los/as entrevistados/as.

- Adquirir herramientas para desarrollar investigaciones donde «los chavos se sientan tomados en cuenta, conociendo su lekil kuxlejal (buen vivir)».

- Conocer nuevas formas de comunicación con el uso de tecnología.

- Llevar a cabo acciones de gestión comunitaria.

n Conocer la importancia de la comunicación en el proceso de trabajo grupal.

- Conocer estrategias para promover la participación de los/as jóvenes en los procesos de investigación sobre sus condiciones de vida y sus puntos de vista.

- Contar con la posibilidad de espejearse en otro/a joven y repensar su situación vital y su proyecto de vida.

- Reconocer la vigencia de «usos y costumbres» que anulan la participación juvenil y la toma de decisiones.

- Generar estrategias que incidan en una mejora en la vida de los/as jóvenes. 
En relación con las habilidades y los conocimientos se mencionaron: aprender a escuchar, colaborar en un equipo, ser responsable, desarrollar habilidades de investigación, dirigirse con respeto a las personas entrevistadas y a los miembros del equipo, lograr empatía, sentirse animado trabajando con jóvenes, tomar notas, analizar primero y opinar luego.

Entre las cualidades y las fortalezas, se señaló el contar con conocimientos previos y con experiencias personales tales como:

- Haber trabajado anteriormente en proyectos comunitarios y, en algunos, casos con jóvenes.

- Contar con la experiencia de haberse unido y tener hijos y/o la experiencia de ejercer el derecho a decidir sobre algún aspecto de su vida.

- Ser flexible y adaptarse a los tiempos de otras personas.

- Poseer conocimientos sobre ciertas dinámicas comunitarias, así como ciertas historias personales de uniones a temprana edad.

- Fomentar las relaciones interculturales y de respeto en las comunidades.

— Desarrollar cualidades para contener emocionalmente a los/as jóvenes.

Como debilidades o aspectos a mejorar se indicaron:

n No delimitar bien los tiempos que implicaba el trabajo de campo para compatibilizarlo con otras actividades como el estudio y el trabajo.

• Sentirse rechazada/o cuando algún/a joven no aceptaba ser entrevistado o mostraba desinterés al momento de la entrevista.

- Contar con un mayor presupuesto para garantizar la dedicación completa al trabajo de investigación.

\section{A nivel grupal}

Se valoró la responsabilidad de la participación individual en el cumplimiento de actividades y de tareas. De igual modo, la respetuosa comunicación y el intercambio de estrategias al interior del grupo posibilitaron llevar a cabo las metas comunes. 
Solo se identificó que la integración de un joven investigador al equipo luego de que se creara el grupo dificultó su integración en un primer momento.

Las dificultades generadas por la falta de señal en algunas de las comunidades en las que el equipo desarrolló su trabajo fue un aspecto que a veces no permitió la participación en las reuniones semanales.

\section{A nivel de la organización y de la comunidad}

En opinión del equipo, el proyecto fue de gran utilidad y pertinencia para las comunidades y para los/as jóvenes. Se valoró, en primer término, construir colectivamente conocimiento sobre las condiciones actuales de las uniones tempranas y sobre los problemas asociados a esta experiencia juvenil. En segundo lugar, se observó la importancia de poder incidir sobre algunas de las problemáticas a través de estrategias educativas y lúdicas.

En este sentido, se considera que el planteamiento y el diseño del proyecto fueron muy pertinentes para documentar las causas y las motivaciones de las uniones tempranas y, desde ahí, entender mejor las alternativas para atender la problemática a nivel local, en beneficio de las juventudes indígenas. Tomar en cuenta a los/as jóvenes, escucharlos y compartir con ellos/as permitió abrir un espacio de diálogo valorado por los/as entrevistados. Escucharlos/as seriamente ayudó a equilibrar el balance entre sus necesidades y las necesidades del proceso de investigación.

\section{Conclusiones y recomendaciones}

El conjunto de hallazgos y de resultados permiten observar cómo las desigualdades sociales, de género, generacionales y étnicas se intersecan en una problemática que requiere de acciones integradoras desde el sector educativo, de desarrollo social, de salud, poniendo en el centro y como eje el ejercicio de una mayor agencia juvenil.

Algunos de los problemas que predisponen a las uniones «tempranas» son: la falta de acceso a anticonceptivos durante el noviazgo, tanto por cuestiones ideológicas como por la falta de accesibilidad a personas o a servicios que puedan satisfacer la demanda, si la hubiera; la deserción escolar; la falta de oportunidades laborales, 
particularmente para las mujeres, y de un proyecto de vida que vaya más allá de formar una familia y tener hijos/as. Entre las principales consecuencias figuran: la violencia psicológica, sufrida particularmente por las jóvenes de parte de suegras y de parejas; el embarazo y sus posibles riesgos; la imposibilidad de continuar los estudios, tanto para las mujeres como para los varones; la exigencia de convertirse en proveedor en el caso de los jóvenes; la sujeción juvenil frente a la autoridad de los padres; la mayor vulnerabilidad que enfrentan las jóvenes si se separan.

Si bien las uniones a temprana edad aparecen normalizadas en estos contextos, desde nuestro punto de vista consideramos importante señalar que dichas uniones deben ser consideradas «obligadas», tanto en el caso en que se presenta un embarazo como en aquellos en los cuales las parejas acuerdan iniciar la convivencia. En ambas situaciones, observamos un escaso margen de acción por parte de los/as jóvenes para tomar ciertas decisiones en torno a su vida. Los/as adultos/as siguen teniendo mucha injerencia.

Adoptar el enfoque de la IAP para estudiar las uniones tempranas con un equipo liderado por jóvenes indígenas posibilitó un nuevo acercamiento entre ellos/as y los/as adultos/as, tanto de la organización civil Ideas Chieltik como de las comunidades (autoridades, abuelas/os, madres/padres). Los/as adultos/as han comprobado que los/as jóvenes pueden llevar adelante investigaciones acerca de los problemas que los/as aquejan con una mirada distinta y han constatado que pueden aprender de y con ellos/as, reconociendo su agencia y sus aportaciones en la generación de conocimiento social que favorezca la movilización comunitaria (Ozer, 2017; Powers \& Tiffany, 2006).

Entre los logros del proyecto se destacan:

- Contribuir a la formación y/o al fortalecimiento de jóvenes en investigación-acción participativa.

- Contribuir a visibilizar una problemática juvenil e involucrar a los/as jóvenes para llevar a adelante acciones acordes a las condiciones actuales.

— Escuchar la voz de los/as jóvenes sobre cómo han sido sus vidas.

- Ofrecerles información sobre su derecho a decidir sobre sus vidas. 
• Favorecer la promoción y/o afianzamiento de distintos nivel de liderazgo en los/as participantes.

- Contribuir a la reflexión con conocimientos surgidos de la experiencia.

Dadas las características del proyecto y el tiempo con el que contábamos para desarrollarlo, nos abocamos a documentar y a analizar los relatos que nos ofrecieron jóvenes unidos recientemente, pero es indispensable contar, también, con las voces de jóvenes que no se han unido, para observar con mayor detenimiento las múltiples trayectorias juveniles. Del mismo modo, es muy importante conocer el punto de vista del «mundo adulto local» (padres y madres de familia, autoridades comunitarias, líderes religiosos, organizaciones civiles, docentes, personal de salud) para complementar ambas visiones. Se requiere así mismo generar más investigaciones empíricas con este enfoque para contar con una base más sólida que permita conocer sus aportaciones en la generación de conocimientos tendiente a encontrar soluciones adecuadas a los/as jóvenes (Ozer, 2017).

Sería deseable que la fundación y la organización continúen apoyando este tipo de iniciativas de investigación y que aboguen por estar soportadas por presupuestos adecuados para garantizar mejores condiciones para que los/as jóvenes investigadores/as se dediquen de tiempo completo a este trabajo y no tengan que realizarlo los fines de semana o restándole tiempo a otras actividades de estudio o de trabajo que realizan para poder vivir.

Este trabajo podrá servir para concientizar a varios actores sociales comunitarios sobre algunos de los problemas que predisponen a las uniones «tempranas» y sobre lo indispensable de continuar trabajando intersectorialmente desde el sector educativo, de desarrollo social y de salud para favorecer que los/as jóvenes permanezcan el mayor tiempo posible en la escuela (aun si están embarazadas y luego de ser madres, en el caso de las mujeres); garantizar un acceso efectivo a información, consejería y métodos anticonceptivos, atención segura del embarazo, parto y posparto; prevenir y atender la violencia de género en todas sus formas; incrementar el diálogo con autoridades comunitarias, padres/madres de familia, personal docente y de salud, líderes religiosos; ampliar las oportunidades laborales; y, sobre todo, lograr el empoderamiento juvenil a través de su participación en proyectos de investigación liderados por ellos/as. 


\section{Referencias}

Arias, P. (2014). La etnografía y la perspectiva de género: nociones y escenarios en debate. En C. Oehmichen Bazán (Ed.), La etnografía y el trabajo de campo en las ciencias sociales (pp. 173-194). Ciudad de México, México: Universidad Nacional Autónoma de México.

Ballinas-Urbina, Y., Evangelista García, A., Nazar Beutelpacher, A. y Salvatierra Izabal, B. (2015). Condiciones sociales y comportamientos sexuales de jóvenes de Chiapas. Papeles de Población, 21(83), 253-286. Recuperado de https://rppoblacion.uaemex.mx/article/view/8331

Consejo Nacional de Evaluación de la Política de Desarrollo Social (CONEVAL). (2015). Evolución de las carencias sociales 2015 y su comparativo con la serie 2010-2014. Recuperado de https://www.coneval.org.mx/Medicion/EDP/Paginas/Datos-del-Modulode-Condiciones-Socioeconomicas.aspx

Cruz Salazar, T. (2009). Mudándose a muchacha. La emergencia de la juventud en indígenas migrantes. En G. Freyemuth y S. Meneses Navarro, Sergio (Coords.), De crianzas, jaibas e infecciones. Indígenas del sureste en la migración (pp. 169-212). Ciudad de México, México: Centro de Investigaciones y Estudios Superiores en Antropología Social (CIESAS).

Evangelista García, A. y Kauffer Michel, E. (2007). Jóvenes y VIH/sida. Contextos de vulnerabilidad en comunidades rurales de la región fronteriza de Chiapas. En R. Tinoco, M. E. Martínez y A. Evangelista García (Coords.), Compartiendo saberes sobre VIH/sida en Chiapas (pp. 77-98). Tuxtla Gutiérrez, México: CISC, ISECH, COESPO, UNPFA.

Dietz, G. y Álvarez Veinguer, A. (2014). Reflexividad, interpretación y colaboración en etnografía: un ejemplo desde la antropología de la educación. En C. Oehmichen Bazán (Ed.), La etnografía y el trabajo de campo en las ciencias sociales (pp. 55-89). Ciudad de México, México: Universidad Nacional Autónoma de México. 
Fondo de las Naciones Unidas para la Infancia (UNICEF). (2019).

El matrimonio infantil. Recuperado de

https://www.unicef.org/es/temas/matrimonio-infantil

González Montes, S. (1999). Las «costumbres» de matrimonio en el

México indígena contemporáneo. En México diverso y desigual.

Enfoques sociodemográficos (pp. 87-105). Ciudad de México, México:

El Colegio de México / Sociedad Mexicana de Demografía (SOMEDE).

González Montes, S. y Mojarro Iñiguez, M. (2011). Algunas dimensiones de la nupcialidad y la situación de las mujeres en ocho regiones indígenas de México. En G. Vázquez Sandrin y A. E. Reyna Bernal (Coords.), Retos, problemáticas y políticas de población indígena en México (pp. 181-205). Ciudad de México, México: Universidad Autónoma del Estado de HidalgoPorrúa.

Greene, M. (2019). Una realidad oculta para niñas y adolescentes. Matrimonios uniones infantiles, tempranas y forzadas en América

Latina y el Caribe. Reporte regional. Washington D.C., Estados Unidos: Plan International Américas y UNFPA. Recuperado de http://www.codajic.org/node/4006

Instituto Nacional de Estadística y Geografía (INEGI). (2010). Chiapas. Recuperado de https://www.inegi.org.mx/default.html

International Child Development Initiatives (ICDI). (2019). Youth-led research. Summary report from Ethiopia, Nepal and Pakistan.

Recuperado de https://bit.ly/3kXirn2

Loewenson, R., Laurell, A. C., Hogstedt, C., D’Ambruoso, L. y Shroff, Z. (2014). Investigación-acción participativa en sistemas de salud. Una guía de métodos. Harare, Zimbabue: TARSC, AHPSR, WHO, IDRC Canadá, EQUINET. 
Magliano, M. J. (2015). Interseccionalidad y migraciones: potencialidades y desafíos. Revista de Estudios Feministas, 23(3), 691-712. Recuperado de http://dx.doi.org/10.1590/0104-026X2015v23n3p691

Mindek, D. (2003). Formación y disolución del matrimonio indígena: una revisión crítica. En D. Robichaux (Comp.), El matrimonio en Mesoamérica. Ayer y hoy. Unas miradas antropológicas (pp. 331-360). Ciudad de México, México: Universidad Iberoamericana.

Ozer, E. J. (2017). Youth-Led Paticipatory Action Research: Overview and Potential for Enhancing Adolescent Development. Child Development Perspectives, 11(3), 17-177. https://doi.org/10.1111/cdep.12228

Powers, J. L. y Tiffany, J. S. (2006). Enganging Youth in Participatory Research and Evaluation. Journal Public Health Management Practice, (Suppl), S79-87.

Programa de las Naciones Unidas para el Desarrollo (PNUD). (2014).

México en breve. Recuperado de

https://www.mx.undp.org/content/mexico/es/home/countryinfo.html https://mx.undp.org

Programa de las Naciones Unidas para el Desarrollo (PNUD). (2020). Objetivos de desarrollo sostenible. Recuperado de https://www.mx.undp.org/content/mexico/es/home/sustainabledevelopment-goals.html

Reartes, D. (2014). Migración y sexualidad. Procesos migratorios, jóvenes indígenas e implicaciones en la sexualidad y la reproducción en los Altos de Chiapas. Rosario, Argentina: Prohistoria ediciones / Centro de Estudios Superiores de México y Centroamérica (CESMECA).

Rivera, E. y Palma, J. L. (2017). Resumen Ejecutivo sobre uniones tempranas. Ciudad de México, México: Investigación en Salud y Demografía (INSAD). 
Rodríguez, G. (2000). Sexualidad y cortejo: contrastes de género y generacionales en una comunidad rural (Tesis de Maestría).

Escuela Nacional de Antropología e Historia (ENAH), Instituto Nacional de Antropología e Historia (INAH), México.

Rodríguez, G. y De Keijzer, B. (2002). La noche se hizo para los hombres.

Sexualidad en los procesos de cortejo entre jóvenes campesinas y campesinos. Ciudad de México, México: The Population Council.

Riaño, Y. (2012). La producción de conocimiento como «minga» y las barreras a la equidad en el proceso investigativo. En S. Corona Berkin y O. Kaltmeier (Coords.), Diálogo. Metodologías horizontales en ciencias sociales y culturas (pp. 137-160). Barcelona, España: Gedisa.

Salles, V. y Tuirán, R. (1997). Dentro del laberinto: salud reproductiva y sociedad. Estudios Demográficos y Urbanos, 12(1 y 2), 11-68.

Valdés, T., Benavente, M. C. y Gysling, J. (1999). El poder en la pareja, la sexualidad y la reproducción. Mujeres de Santiago. Santiago de Chile, Chile: Facultad Latinoamericana de Ciencias Sociales (FLACSO).

\section{Notas}

1 Organización civil que actúa en Chiapas, México, desde 2009. Su misión es «contribuir al desarrollo integral y al bienestar de los jóvenes indígenas a través de talleres educativos, culturales y artísticos» (Ch'ieltik, 2020).

2 El equipo estuvo formado por Eliber Gómez Abadía, Laura Pérez González, Jaider García Vásquez, Luz Clarita Castañeda Ramos, Guadalupe Pérez Sántiz y Luis Cruz Mendoza.

3 «[...] empowers children and young people growing up in difficult circumstances, to understand better their communities and the social values and issues tha involve and affect them so they become active agents of change» (ICDI, 2019, p. 5).

4 «1. Support girls and boys in understanding the world they liven in, 2. give children the necessary sills to make decisions, 3. support children to become agents of change in their communities» (ICDI, 2019, p. 6). 
5 Benefits for children: 1. Improved capacity to make choices, reflect and think critically. 2. Better writing, commucation and organizational skills, 3. More confidence to contribute to improving children's lives in their communities, 4. Bring valuable insights and original contributions to kwoledge, 5. Observe with different eyes, ask different questions, have different concerns. 